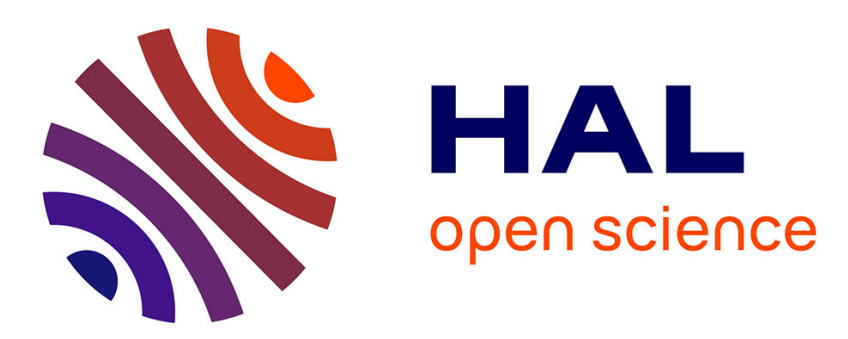

\title{
Polynomial Estimation of Time-varying Multipath Gains with ICI Mitigation in OFDM Systems
}

\author{
Hussein Hijazi, Laurent Ros
}

\section{To cite this version:}

Hussein Hijazi, Laurent Ros. Polynomial Estimation of Time-varying Multipath Gains with ICI Mitigation in OFDM Systems. ISCCSP 2008 - 3rd International Symposium on Communications, Control and Signal Processing, Mar 2008, St-Julians, Malta. 5 p. hal-00275606

\section{HAL Id: hal-00275606 https://hal.science/hal-00275606}

Submitted on 24 Apr 2008

HAL is a multi-disciplinary open access archive for the deposit and dissemination of scientific research documents, whether they are published or not. The documents may come from teaching and research institutions in France or abroad, or from public or private research centers.
L'archive ouverte pluridisciplinaire HAL, est destinée au dépôt et à la diffusion de documents scientifiques de niveau recherche, publiés ou non, émanant des établissements d'enseignement et de recherche français ou étrangers, des laboratoires publics ou privés. 


\title{
Polynomial Estimation of Time-varying Multipath Gains with ICI Mitigation in OFDM Systems
}

\author{
Hussein Hijazi and Laurent Ros \\ GIPSA-lab, Departement Image Signal \\ BP 46 - 38402 Saint Martin d'Hères - FRANCE \\ E-mail: hussein.hijazi@gipsa-lab.inpg.fr, laurent.ros@gipsa-lab.inpg.fr
}

\begin{abstract}
In this paper, we present a Mean Square Error (MSE) theoretical analysis for a multipath channel complex gains estimation algorithm with inter-sub-carrier-interference (ICI) mitigation in orthogonal frequency division multiplexing (OFDM) high speed mobile receiver. Each complex gain timevariation is approximated in a polynomial fashion within several OFDM symbols, where the polynomial coefficients are obtained from the estimated time-averaged gain values. After that, the channel matrix is easily computed and the ICI is reduced by using successive interference suppression (SIS) during data symbol detection. The algorithm performance is further enhanced by an iterative procedure. Theoretical analysis for Rayleigh channel with Jakes'spectrum and simulation results show that the low computational complexity proposed algorithm has good performance in the presence of high normalised Doppler spread.
\end{abstract}

Index Terms-OFDM, Time-varying channels, Polynomial approximation, Mean square error

\section{INTRODUCTION}

ORTHOGONAL frequency division multiplexing (OFDM) is an attractive technique for high-speed data transmission in mobile communication [7]. Assuming insertion of pilottones (called comb-type pilot) into each OFDM symbol, the conventional channel estimation methods consist generally of estimating the channel at pilot frequencies and next interpolating the channel frequency response. The channel estimation at the pilot frequencies can be based on Least Square (LS) criterion, or Linear Minimum Mean-Square-Error (LMMSE) criterion for better performance [8]. In [9], low-pass interpolation (LPI) has been shown to perform better than all interpolation techniques used in channel estimation.

For fast time-varying channel, many existing works resort to estimate the equivalent discrete-time channel taps which are modeled by the basis expansion model (BEM) [3]. The BEM methods [3] are Karhunen-Loeve BEM (KL-BEM), prolate spheroidal BEM (PS-BEM), complex exponential BEM (CE$\mathrm{BEM})$ and polynomial BEM (P-BEM). A great deal of attention has been paid to the P-BEM [4], although its modeling performance is rather sensitive to the Doppler spread; nevertheless, it provides a better fit for low, than for high Doppler spreads.

Our interesting is to estimate directly the physical channel instead of the equivalent discrete-time channel taps. That means estimating the physical propagation parameters such as multipath delays and multipath complex gains. In [1], we have proposed an algorithm for channel matrix estimation and inter-sub-carrier-interference (ICI) reduction whose execution is done per block of OFDM symbols. Assuming the availability of delay information, the complex gains time-variation within one OFDM symbol were obtained by interpolating the complex gains time average estimated over each symbol of the block. This algorithm (without ICI suppression) performs better than the conventional methods and becomes better with starting ICI suppression, but with high computational complexity.

In this paper, we present a new low-complexity iterative algorithm for complex gains estimation with ICI mitigation using comb-type pilot. By exploiting the nature of the channel, the delays are assumed invariant and perfectly estimated as we have already done in OFDM [1] and CDMA [2] contexts. Notice that an initial very performant multipath time delays estimation can be obtained by using the ESPRIT (estimation of signal parameters by rotational invariance techniques) method [10] [11]. First, we compute the complex gains time average over the effective duration of the OFDM symbol by using LS criterion as we have already done in [1]. Then, we show that each complex gain time-variation can be approximated in a polynomial fashion within several OFDM symbols where the coefficients of each polynomial are calculated from the estimated time average values. Hence, thanks to the polynomial modeling, the channel matrix can be computed with low complexity from the estimated coefficients and the ICI is reduced using SIS in data symbol detection. The present proposed algorithm has demonstrated a great improvement in performance while reducing complexity as compared to the one presented in [1] thanks to an iterative procedure. Moreover, we give a theoretical Mean Square Error (MSE) analysis (including lower bound computation) of our channel estimation algorithm in terms of the normalised (by the OFDM symbol-time) Doppler spread. This further demonstrates the effectiveness of the proposed algorithm. This paper is organized as follows. Section II introduces the OFDM system model and section III the polynomial modeling. Section IV presents polynomial coefficients estimation and iterative algorithm. Next, Section V gives some simulation results. We conclude the paper in Section VI.

Notation: The notations used in this paper are as follows. Upper (lower) bold face letters denote matrices (column 
vectors). $[\mathbf{x}]_{k}$ denotes the $k$ th element of the vector $\mathbf{x}$, and $[\mathbf{X}]_{k, m}$ denotes the $[k, m]$ th element of the matrix $\mathbf{X} . \mathbf{I}_{N}$ is a $N \times N$ identity matrix and $\operatorname{diag}\{\mathbf{x}\}$ is a diagonal matrix with $\mathbf{x}$ on its main diagonal. The superscripts $(\cdot)^{T}$ and $(\cdot)^{H}$ stand respectively for transpose and Hermitian operators. $|\cdot|, \operatorname{Tr}(\cdot)$ and $\mathrm{E}[\cdot]$ are respectively the determinant, trace and expectation operations, and $\operatorname{Re}(\cdot),\|\cdot\|$ and $(\cdot)^{*}$ are respectively the real part, magnitude and conjugate of a complex number or matrix. $\|\mathbf{X}\|^{2}$ is the Frobenius matrix norm and $J_{0}(\cdot)$ denotes the zeroth-order Bessel function of the first kind.

\section{OFDM SYSTEM MODEL}

We denote the number of subcarriers by $\mathrm{N}$ and the sampling time by $T_{s}$. The duration of an OFDM symbol is $T=v T_{s}$ with $v=N+N_{g}$ where $N_{g}$ is the length of the cyclic prefix. In an OFDM system, the transmitter usually applies an $N$-point IFFT to a data block normalized QAM-symbols $\left\{x_{(n)}[b]\right\}$ (i.e., $\mathrm{E}\left[x_{(n)}[b] x_{(n)}[b]^{*}\right]=1$ ) and adds a cyclic prefix $(\mathrm{CP})$ as $s_{(n)}[q]=\frac{1}{N} \sum_{b=-\frac{N}{2}}^{\frac{N}{2}-1} x_{(n)}[b] e^{j 2 \pi \frac{b q}{N}}$, where $n$ and $b$ represent respectively the OFDM symbol index and the subcarrier index and $q \in\left[-N_{g}, N-1\right]$. The output baseband signal of the transmitter is sent over a multipath Rayleigh fading channel characterized by:

$$
h(t, \tau)=\sum_{l=1}^{L} \alpha_{l}(t) \delta\left(\tau-\tau_{l} T_{s}\right)
$$

where $L$ is the total number of propagation paths, $\alpha_{l}$ is the $l$ th complex gains of variance $\sigma_{\alpha_{l}}^{2}$ and $\tau_{l}$ is the $l$ th delay normalized by the sampling time ( $\tau_{l}$ is not necessarily an integer). $\left\{\alpha_{l}(t)\right\}$ are wide-sense stationary (WSS) narrowband complex Gaussian processes with the so-called Jakes' power spectrum of maximum Doppler frequency $f_{d}$ [5] and uncorellated with respect to each other. The average energy of the channel is normalized to one (i.e., $\sum_{l=1}^{L} \sigma_{\alpha_{l}}^{2}=1$ ).

At the receiver side, after passing to discrete time through low pass filtering and A/D conversion, the $\mathrm{CP}$ is removed assuming that its length is no less than the maximum delay. Afterwards, a $N$-point FFT is applied to transform the sequence into frequency domain. If we consider that the $N$ transmission subcarriers are within the flat region of the frequency response of each of the transmitter and receiver filters, then, omitting the index time $n$, the $\mathrm{N}$ received subcarriers are given by [1]:

$$
\mathbf{y}=\mathbf{H} \mathbf{x}+\mathbf{w}
$$

where $\mathbf{x}, \mathbf{y}, \mathbf{w}$ are $N \times 1$ vectors given by:

$$
\begin{aligned}
\mathbf{x} & =\left[x\left[-\frac{N}{2}\right], x\left[-\frac{N}{2}+1\right], \ldots, x\left[\frac{N}{2}-1\right]\right]^{T} \\
\mathbf{y} & =\left[y\left[-\frac{N}{2}\right], y\left[-\frac{N}{2}+1\right], \ldots, y\left[\frac{N}{2}-1\right]\right]^{T} \\
\mathbf{w} & =\left[w\left[-\frac{N}{2}\right], w\left[-\frac{N}{2}+1\right], \ldots, w\left[\frac{N}{2}-1\right]\right]^{T}
\end{aligned}
$$

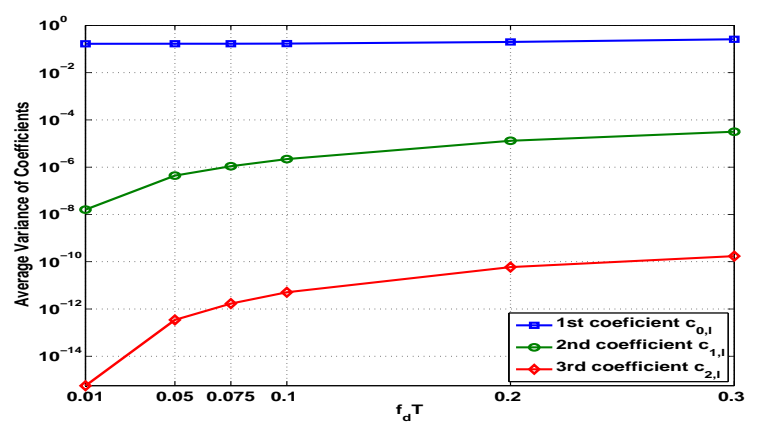

Fig. 1. The average variance of each coefficient for a normalized channel of $L=6$ paths and $N_{c}=3$

and $\mathbf{H}$ is a $N \times N$ channel matrix with elements given by:

$$
[\mathbf{H}]_{k, m}=\frac{1}{N} \sum_{l=1}^{L}\left[e^{-j 2 \pi\left(\frac{m-1}{N}-\frac{1}{2}\right) \tau_{l}} \sum_{q=0}^{N-1} \alpha_{l}\left(q T_{s}\right) e^{j 2 \pi \frac{m-k}{N} q}\right]
$$

where $\left\{\alpha_{l}\left(q T_{s}\right)\right\}$ is the $T_{s}$ spaced sampling of the $l$ th complex gain and $w[b]$ is white complex Gaussian noise with variance $\sigma^{2}$. The channel matrix contains the time average of the channel frequency response $[\mathbf{H}]_{k, k}$ on its diagonal and the coefficients of ICI $[\mathbf{H}]_{k, m}$ for $k \neq m$.

\section{Complex Gain Polynomial Modeling}

In this section, we show that, for high realistic doppler spread $f_{d} T$, each sampled complex gain $\boldsymbol{\alpha}_{l}=$ $\left[\alpha_{l}\left(-N_{g} T_{s}\right), \ldots, \alpha_{l}\left(\left(v N_{c}-N_{g}-1\right) T_{s}\right)\right]^{T}$ within $N_{c}$ OFDM symbols can be approximated with a polynomial model of $N_{c}$ coefficients $\mathbf{c}_{l}=\left[c_{0, l}, \ldots, c_{N_{c}-1, l}\right]^{T}$. Thus, for $q \in \mathcal{D}=$ $\left[-N_{g}, v N_{c}-N_{g}-1\right], \alpha_{l}\left(q T_{s}\right)$ can be expressed as: $\alpha_{l}\left(q T_{s}\right)=$ $\sum_{d=0}^{N_{c}-1} c_{d, l} q^{d}+\xi_{l}[q]$, where $\xi_{l}[q]$ is the model error. We also show that a good approximation can be obtained by calculating the $N_{c}$ coefficients from only $\overline{\boldsymbol{\alpha}}_{l}=\left[\bar{\alpha}_{l, 0}, \ldots, \bar{\alpha}_{l, N_{c}-1}\right]^{T}$, where $\bar{\alpha}_{l, d}=\frac{1}{N} \sum_{q=d v}^{d v+N-1} \alpha_{l}\left(q T_{s}\right)$ is the time average over the effective duration of the $(d+1)$ th OFDM symbol of the $l$ th complex gain.

Optimal Polynomial: The optimal polynomial $\boldsymbol{\alpha}_{\text {opt }_{l}}$, which is least-squares fitted (linear and polynomial regression) [6] to $\boldsymbol{\alpha}_{l}$, and its $N_{c}$ coefficients $\mathbf{c}_{\text {opt }_{l}}$ are given by:

$$
\begin{aligned}
\boldsymbol{\alpha}_{\mathbf{o p t}_{l}} & =\mathbf{Q}^{T} \mathbf{c}_{\mathbf{o p t}_{l}}=\mathbf{S} \boldsymbol{\alpha}_{l} \\
\mathbf{c}_{\mathbf{o p t}_{l}} & =\left(\mathbf{Q} \mathbf{Q}^{T}\right)^{-1} \mathbf{Q} \boldsymbol{\alpha}_{l}
\end{aligned}
$$

where $\mathbf{Q}$ is a $N_{c} \times v N_{c}$ matrix of elements $[\mathbf{Q}]_{k, m}=(m-$ $\left.N_{g}-1\right)^{(k-1)}$ and $\mathbf{S}=\mathbf{Q}^{T}\left(\mathbf{Q} \mathbf{Q}^{T}\right)^{-1} \mathbf{Q}$ is a $v N_{c} \times v N_{c}$ matrix. It provides the MMSE approximation for all polynomials containing Nc coefficients, given by:

$$
\begin{aligned}
\operatorname{MMSE}_{l} & =\frac{1}{v N_{c}} \mathrm{E}\left[\left(\boldsymbol{\alpha}_{l}-\boldsymbol{\alpha}_{\mathbf{o p t}_{l}}\right)^{H}\left(\boldsymbol{\alpha}_{l}-\boldsymbol{\alpha}_{\mathbf{o p t}_{l}}\right)\right] \\
& =\frac{1}{v N_{c}} \operatorname{Tr}\left(\left(\mathbf{I}_{v N_{c}}-\mathbf{S}\right) \mathbf{R}_{\boldsymbol{\alpha}_{l}}\left(\mathbf{I}_{v N_{c}}-\mathbf{S}^{T}\right)\right)
\end{aligned}
$$




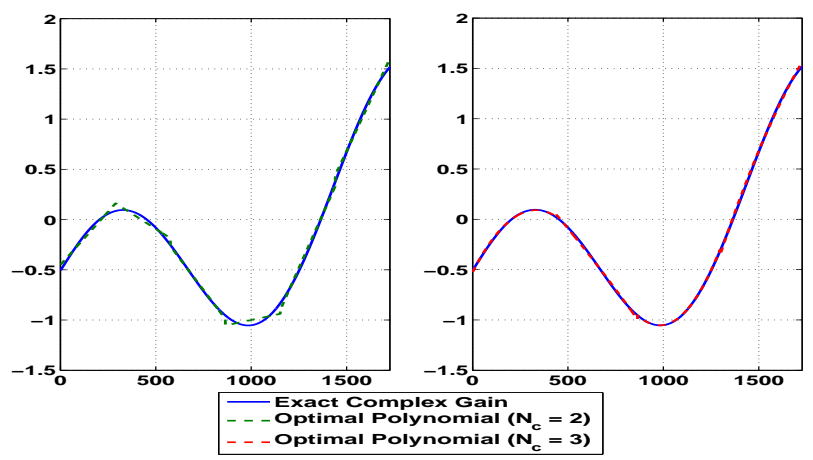

Fig. 2. The real part of the exact complex gain and its optimal polynomial modeling for one path channel realization over 12 OFDM symbols with $f_{d} T=0.1$ and $N_{c}=2$ (left) and 3 (right)

where $\mathbf{R}_{\boldsymbol{\alpha}_{l}}=\mathrm{E}\left[\boldsymbol{\alpha}_{l} \boldsymbol{\alpha}_{l}^{H}\right]$ is the $v N_{c} \times v N_{c}$ correlation matrix of $\boldsymbol{\alpha}_{l}$ of elements given by:

$$
\left[\mathbf{R}_{\boldsymbol{\alpha}_{l}}\right]_{k, m}=\sigma_{\alpha_{l}}^{2} J_{0}\left(2 \pi f_{d} T_{s}(k-m)\right)
$$

The $N_{c}$ coefficients $\mathbf{c}_{\mathbf{o p t}}$ are correlated complex gaussian variables with zero-means and covariance matrix given by:

$$
\mathbf{R}_{\mathbf{c}_{\mathbf{o p t}_{l}}}=\mathrm{E}\left[\mathbf{c}_{\mathbf{o p t}_{l}} \mathbf{c}_{\mathbf{o p t}_{l}}{ }^{H}\right]=\left(\mathbf{Q} \mathbf{Q}^{T}\right)^{-1} \mathbf{Q R}_{\boldsymbol{\alpha}_{l}} \mathbf{Q}^{T}\left(\mathbf{Q} \mathbf{Q}^{T}\right)^{-1}
$$

Fig. 1 shows the average (over $L=6$ paths) variance of each coefficient for $N_{c}=3$. We notice that the variance decreases very quickly in terms of number of coefficients. That means the second and the third coefficients are very small.

Desired Polynomial: We now aim to find the polynomial approximation of $N_{c}$ coefficients by knowing only $\overline{\boldsymbol{\alpha}}_{l}$. This polynomial and its coefficients are given by:

$$
\boldsymbol{\alpha}_{\mathbf{d e s}_{l}}=\mathbf{Q}^{T} \mathbf{c}_{\text {des }_{l}}=\mathbf{V} \overline{\boldsymbol{\alpha}}_{l} \quad \text { and } \quad \mathbf{c}_{\mathbf{d e s}_{l}}=\mathbf{T}^{-1} \overline{\boldsymbol{\alpha}}_{l}
$$

where $\mathbf{T}$ is the transfer matrix between $\mathbf{c}_{\mathbf{d e s}_{l}}$ and $\overline{\boldsymbol{\alpha}}_{l}$, and $\mathbf{V}=$ $\mathbf{Q}^{T} \mathbf{T}^{-1}$. For $N_{c}=3, \mathbf{T}$ is given by:

$$
\mathbf{T}=\left[\begin{array}{ccc}
1 & \frac{N-1}{2} & \frac{(N-1)(2 N-1)}{6} \\
1 & \frac{N-1}{2}+v & \frac{(N-1)(2 N-1)}{6}+(N-1) v+v^{2} \\
1 & \frac{N-1}{2}+2 v & \frac{(N-1)(2 N-1)}{6}+2(N-1) v+4 v^{2}
\end{array}\right]
$$

Note that, for $N_{c}=2$, the transfer matrix will be the $2 \times 2$ upper block matrix in the top-left corner of the matrix $\mathbf{T}$ with $N_{c}=3$.

The MSE of this polynomial approximation is given by:

$$
\begin{gathered}
\mathrm{MSE}_{\mathrm{des}_{l}}=\frac{1}{v N_{c}} \mathrm{E}\left[\mathbf{e}_{\operatorname{des}_{l}} \mathbf{e}_{\operatorname{des}_{l}}^{H}\right]= \\
\frac{1}{v N_{c}} \operatorname{Tr}\left(\mathbf{R}_{\boldsymbol{\alpha}_{l}}+\mathbf{V} \mathbf{R}_{\overline{\boldsymbol{\alpha}}_{l}} \mathbf{V}^{T}-\mathbf{R}_{\boldsymbol{\alpha}_{l} \overline{\boldsymbol{\alpha}}_{l}} \mathbf{V}^{T}-\mathbf{V} \mathbf{R}_{\boldsymbol{\alpha}_{l} \overline{\boldsymbol{\alpha}}_{l}}^{H}\right)
\end{gathered}
$$

where $\mathbf{e}_{\mathbf{d e s}_{l}}=\boldsymbol{\alpha}_{l}-\boldsymbol{\alpha}_{\mathbf{d e s}_{l}}, \mathbf{R}_{\overline{\boldsymbol{\alpha}}_{l}}$ is the correlation matrix of $\overline{\boldsymbol{\alpha}}_{l}$ and $\mathbf{R}_{\boldsymbol{\alpha}_{l} \overline{\boldsymbol{\alpha}}_{l}}$ is the cross-correlation matrix between $\boldsymbol{\alpha}_{l}$ and $\overline{\boldsymbol{\alpha}}_{l}$ with elements given by:

$$
\begin{gathered}
{\left[\mathbf{R}_{\overline{\boldsymbol{\alpha}}_{l}}\right]_{k, m}=\frac{\sigma_{\alpha_{l}}^{2}}{N^{2}} \sum_{\substack{q_{1}=k v-v \\
k v-N_{g}-1}}^{m q_{2}=m v-v} J_{0}\left(2 \pi f_{d} T_{s}\left(q_{1}-q_{2}\right)\right)} \\
{\left[\mathbf{R}_{\boldsymbol{\alpha}_{l} \overline{\boldsymbol{\alpha}}_{l}}\right]_{k, m}=\frac{\sigma_{\alpha_{l}}^{2}}{N} \sum_{q=m v-v}^{m v+N_{g}-1} J_{0}\left(2 \pi f_{d} T_{s}\left(k-q-N_{g}-1\right)\right)}
\end{gathered}
$$

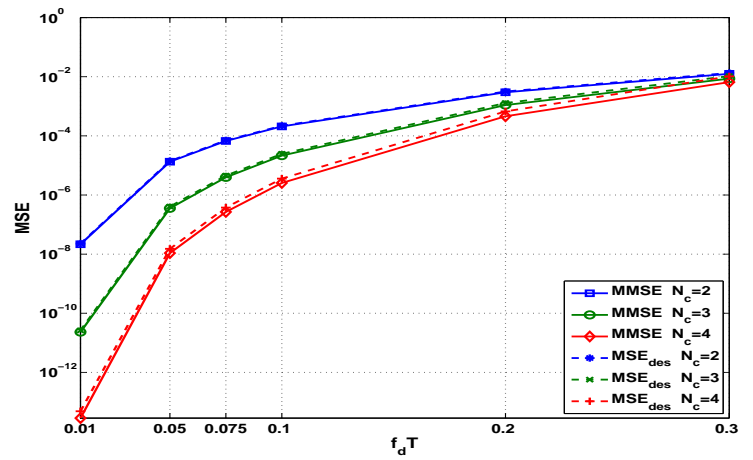

Fig. 3. Comparison between MMSE and $\mathrm{MSE}_{\text {des }}$ for a normalized channel with $L=6$ paths

From Fig. 2, it is observed that we have a good polynomial approximation. As we see in Fig. 3, we have MSE $\mathrm{des}_{\mathrm{d}} \approx \mathrm{MMSE}$ and for $f_{d} T \leq 0.1, \mathrm{MSE}_{\mathrm{des}} \leq 10^{-4}$, even with just $N_{c}=2$ coefficients. This proves that, for high realistic $f_{d} T$, we can approximate $\boldsymbol{\alpha}_{l}$ with a polynomial model of $N_{c}$ coefficients and we can calculate the polynomial approximation which approaches the MMSE approximation from only the time average values $\overline{\boldsymbol{\alpha}}_{l}$. Under this polynomial approximation, the channel matrix (see equation (3)) for the $n$th symbol of $N_{c}$ OFDM symbols can be simply defined as:

$\mathbf{H}_{(n)}=\frac{1}{N} \sum_{d=0}^{N_{c}-1} \mathbf{B}_{(n, d)} \quad$ with $\quad \mathbf{B}_{(n, d)}=\mathbf{M}_{(n, d)} \operatorname{diag}\left\{\mathbf{F} \boldsymbol{\chi}_{d}\right\}$

where $\chi_{d}=\left[c_{d, 1}, \ldots, c_{d, L}\right]^{T}, \mathbf{F}$ is the $N \times L$ Fourier matrix and $\mathbf{M}_{(n, d)}$ is a $N \times N$ matrix given by:

$$
\begin{aligned}
{[\mathbf{F}]_{k, m} } & =e^{-j 2 \pi\left(\frac{k-1}{N}-\frac{1}{2}\right) \tau_{m}} \\
{\left[\mathbf{M}_{(n, d)}\right]_{k, m} } & =\sum_{q=0}^{N-1}(q+(n-1) v)^{d} e^{-j 2 \pi \frac{m-k}{N} q}
\end{aligned}
$$

where $n \in\left[1, N_{c}\right]$. Note that the terms of the matrix $\mathbf{M}_{(n, d)}$ can be easily computed and stored by using the properties of power series.

\section{Polynomial Coefficients Estimation And ITERATIVE ALGORITHM}

In this section, we propose a method based on comb-type pilots and multipath time delays information to estimate the $N_{c}$ coefficients $\mathbf{c}_{\text {des }_{l}}$ of the polynomial approximation $\boldsymbol{\alpha}_{\mathbf{d e s}_{l}}$ for each path.

\section{A. Pilot Pattern and Estimation of Polynomial Coefficients}

The $N_{p}$ pilot subcarriers are evenly inserted into the $N$ subcarriers at the positions $\mathcal{P}=\left\{p_{s} \mid p_{s}=(s-1) L_{f}+\right.$ $\left.1, s=1, \ldots, N_{p}\right\}$ with $L_{f}$ the distance between two adjacent pilots. As we will see with equation (15), $N_{p}$ must fulfill the following requirement: $N_{p} \geq L$. The received pilot subcarriers can be written as the sum of three components:

$$
\mathbf{y}_{\mathbf{p}}=\operatorname{diag}\left\{\mathbf{x}_{\mathbf{p}}\right\} \mathbf{F}_{\mathbf{p}} \boldsymbol{a}+\mathbf{H}_{\mathbf{p}} \mathbf{x}+\mathbf{w}_{\mathbf{p}}
$$


where $\mathbf{x}_{\mathbf{p}}, \mathbf{y}_{\mathbf{p}}$ and $\mathbf{w}_{\mathbf{p}}$ are $N_{p} \times 1$ vectors, $\mathbf{H}_{\mathbf{p}}$ is a $N_{p} \times N$ matrix, $\boldsymbol{a}=\left[\bar{\alpha}_{1}, \ldots, \bar{\alpha}_{L}\right]^{T}$ and $\mathbf{F}_{\mathbf{p}}$ is the $N_{p} \times L$ Fourier transform matrix with elements given by:

$$
\overline{\alpha_{l}}=\frac{1}{N} \sum_{q=0}^{N-1} \alpha_{l}\left(q T_{s}\right) \quad \text { and } \quad\left[\mathbf{F}_{\mathbf{p}}\right]_{k, l}=e^{-j 2 \pi \frac{p_{k}}{N} \tau_{l}}
$$

The first component of (13) is the desired term without ICI and the second component is the ICI term. By neglecting the ICI contribution, the LS-estimator of $\boldsymbol{a}$ is [1]:

$$
a_{\mathbf{L S}}=\mathbf{G y}_{\mathbf{p}}
$$

where $\mathbf{G}=\left(\mathbf{F}_{\mathbf{p}}^{H} \operatorname{diag}\left\{\mathbf{x}_{\mathbf{p}}\right\}^{H} \operatorname{diag}\left\{\mathbf{x}_{\mathbf{p}}\right\} \mathbf{F}_{\mathbf{p}}\right)^{-1} \mathbf{F}_{\mathbf{p}}^{H} \operatorname{diag}\left\{\mathbf{x}_{\mathbf{p}}\right\}^{H}$. Estimating $\boldsymbol{a}$ for $N_{c}$ consecutive OFDM symbols, the $N_{c}$ polynomial coefficients of each complex gains are obtained (section 3) by:

$$
\hat{\mathbf{C}}_{\text {des }}=\mathbf{T}^{-1} \mathbf{A}_{\mathbf{L S}}
$$

where $\hat{\mathbf{C}}_{\text {des }}=\left[\hat{\mathbf{c}}_{\text {des }_{1}}, \ldots, \hat{\mathbf{c}}_{\text {des }_{L}}\right]$ and $\mathbf{A}_{\mathbf{L S}}=\left[\overline{\boldsymbol{\alpha}}_{\mathbf{L S}}, \ldots, \overline{\boldsymbol{\alpha}}_{\mathbf{L S}}\right]$ are $N_{c} \times L$ matrices.

\section{B. Iterative Algorithm}

In the iterative algorithm, the OFDM symbols are grouped in blocks of $N_{c}$ OFDM symbols each one. The algorithm execution is done in two stages: initialization stage (IS) and sliding stage (SS). IS applies only to the first received block (i.e. $n=1, \ldots, N_{c}$ ) and SS applies to each following OFDM symbol (i.e. $n>N_{c}$ ) while benefitting from $\left(N_{c}-1\right)$ preceding time averages complex gains estimated with ICI reduced. IS and SS proceed as following:

$$
\begin{aligned}
& \text { initialization: } \\
& i \leftarrow 1 \\
& \text { if }(\text { IS }) ; \\
& \mathbf{Y}_{\mathbf{p}_{(i)}}=\left[\mathbf{y}_{\mathbf{p}_{(1, i)}}, \ldots, \mathbf{y}_{\mathbf{p}_{\left(N_{c}, i\right)}}\right] \text { where } \mathbf{y}_{\mathbf{p}_{(n, i)}}=\mathbf{y}_{\mathbf{p}_{(n)}} n=1, \ldots, N_{c} \\
& \text { elseif }(\mathrm{SS}) ; \\
& n \leftarrow n+1 \\
& \left\{\left[\mathbf{A}_{\mathbf{L S}}\right]_{k, m}, k=1, . ., N_{c}-1\right\}=\left\{\left[\mathbf{A}_{\mathbf{L S}}\right]_{k, m}, k=2, . ., N_{c}\right\} \\
& \mathbf{y}_{\mathbf{p}_{(n, i)}}=\mathbf{y}_{\mathbf{p}_{(n)}}
\end{aligned}
$$

recursion :

1) if (IS); $\mathbf{A}_{\mathbf{L S}}^{T}=\mathbf{G} \mathbf{Y}_{\mathbf{p}_{(i)}}$ elseif $(\mathrm{SS}) ; \quad \boldsymbol{a}_{\mathbf{L S}}=\mathbf{G y}_{\mathbf{p}_{(n, i)}}$ $\left\{\left[\mathbf{A}_{\mathbf{L S}}\right]_{N_{c}, m}, m=1, . ., L\right\}=\left\{\left[\boldsymbol{a}_{\mathbf{L S}}\right]_{m}, m=1, . ., L\right\}$

2) $\hat{\mathbf{C}}_{\text {des }}=\mathbf{T}^{-1} \mathbf{A}_{\mathbf{L S}}$

3) compute the channel matrix using (11) if (IS); $\hat{\mathbf{H}}_{(n, i)} n=1, \ldots, N_{c}$ elseif (SS); $\hat{\mathbf{H}}_{\left(N_{c}, i\right)}$

4) remove the pilot ICI from the received data sub-carriers $\mathbf{y}_{\mathbf{d}_{(n)}}$

5) detection of data symbols $\hat{\mathbf{x}}_{\mathbf{d}_{(n, i)}}$

6) $\mathbf{y}_{\mathbf{p}_{(n, i+1)}}=\mathbf{y}_{\mathbf{p}_{(n)}}-\hat{\mathbf{H}}_{\mathbf{p}_{(n, i)}} \hat{\mathbf{x}}_{(n, i)}$

7) $i \stackrel{\leftarrow}{\leftarrow}+1$ where $i$ represents the iteration number. The data symbols detection in step 5 are estimated by SIS scheme with the optimal ordering and one tap frequency equalizer [1]. Notice that at the end of IS, $n=N_{c}$.

\section{Computational Complexity}

We now aim to find the implementation complexity in term of number of multiplication for the sliding stage. The matrices $\mathbf{F}, \mathbf{F}_{\mathbf{p}}, \mathbf{G}, \mathbf{T}^{-1}$ and $\mathbf{M}_{(n, d)}$ are pre-computed and stored if the pilot subcarriers are fixed and the delays are invariant for a great number of OFDM symbols. The complexity for LS-estimator of $\boldsymbol{a}$ in step 1 is $L \times N_{p}$ and for estimation of $N_{c}$ polynomial coefficients in step 2 is $L \times N_{c}^{2}$. The computation cost of computing the channel matrix $\mathbf{H}_{(n)}$ in step 3 is $N N_{c}(N+L)$, less than that in [1] which is $L N^{2}(N+1)$. The complexity of removing the ICI in step 4,5 and 6 is $N_{p}\left(N-N_{p}\right)+\frac{\left(N-N_{p}\right)\left(N-N_{p}+1\right)}{2}+N_{p}(N-1)$.

\section{Mean Square Error (MSE) Analysis}

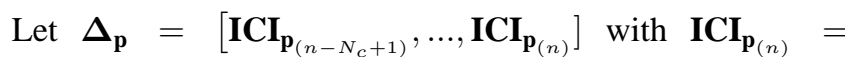
$\mathbf{H}_{\mathbf{p}_{(n)}} \mathbf{x}_{(n)}$ is the ICI for the $n$th symbol of $N_{c}$ OFDM symbols. The error of the estimator of $\boldsymbol{a}$ over $N_{c}$ OFDM symbols is defined as $\mathcal{E}=\hat{\mathbf{A}}^{T}-\mathbf{A}^{T}$. The error between the $l$ th exact complex gain $\boldsymbol{\alpha}_{l}$ and the $l$ th estimated polynomial $\hat{\boldsymbol{\alpha}}_{\mathbf{d e s}_{l}}$ is given by:

$$
\mathbf{e}_{l}=\boldsymbol{\alpha}_{l}-\mathbf{V} \hat{\overline{\boldsymbol{\alpha}}}_{l}=\mathbf{e}_{\mathbf{d e s}_{l}}-\mathbf{V} \boldsymbol{\epsilon}_{l}
$$

where $\epsilon_{l}^{T}$ is the $l$ th row of the matrix $\mathcal{E}$. So the MSE between the $l$ th exact complex gain and the $l$ th estimated polynomial is given by:

$$
\begin{aligned}
& \mathrm{MSE}_{l}=\frac{1}{v N_{c}} \mathrm{E}\left[\mathbf{e}_{l}^{H} \mathbf{e}_{l}\right] \\
& =\mathrm{MSE}_{\mathrm{des}_{l}}+\frac{1}{v N_{c}} \mathrm{E}\left[\boldsymbol{\epsilon}_{l}^{H} \mathbf{V}^{H} \mathbf{V} \boldsymbol{\epsilon}_{l}\right] \\
& -\frac{2}{v N_{c}} \operatorname{Re}\left(\mathrm{E}\left[\mathbf{e}_{\operatorname{des}_{l}}^{H} \mathbf{V} \boldsymbol{\epsilon}_{l}\right]\right)
\end{aligned}
$$

If ICI are completely eliminated then, the elements of $\mathcal{E}$ are uncorrelated with respect to each other and to the elements of $\mathbf{e}_{\mathbf{d e s}_{l}}$. Thus, from (18) we have:

$$
\operatorname{MSE}_{l}(\text { without ICI })=\operatorname{MSE}_{\operatorname{des}_{l}}+\frac{\|\mathbf{V}\|^{2}}{v N_{c}} \mathrm{E}\left[[\mathcal{E}]_{l, 1}[\mathcal{E}]_{l, 1}^{*}\right]
$$

Interpreting the right hand side of (19), the first component is the model error, whereas the second component is the MSE of the $l$ th estimated polynomial without ICI. This second component is due to the error of the estimator of $\boldsymbol{a}$ without ICI amplified by a gain $\mathcal{G}=\frac{\|\mathbf{V}\|^{2}}{v N_{c}}$ linked to the polynomial modeling. So, the lower bound (LB) of the estimator of $\boldsymbol{a}$ (without ICI) leads to the LB of $\mathrm{MSE}_{l}$ (without ICI). The Standard CRAMER-RAO BOUND (SCRB) [14] for the estimator of $\boldsymbol{a}$ with known ICI is given by:

$$
\mathbf{S C R B}_{\boldsymbol{a}}=\frac{1}{S N R}\left(\mathbf{F}_{\mathbf{p}}^{H} \operatorname{diag}\left\{\mathbf{x}_{\mathbf{p}}\right\}^{H} \operatorname{diag}\left\{\mathbf{x}_{\mathbf{p}}\right\} \mathbf{F}_{\mathbf{p}}\right)^{-1}
$$




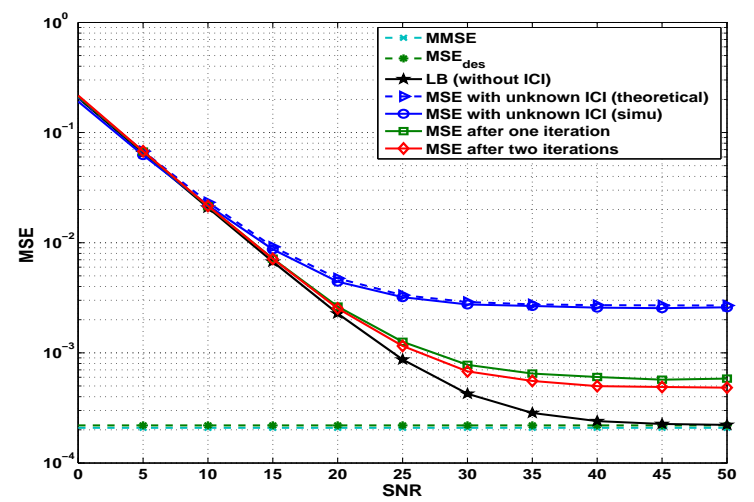

Fig. 4. MSE for $f_{d} T=0.1$ and $N_{c}=2$

where $S N R=\frac{1}{\sigma^{2}}$ is the normalized signal to noise ratio. Hence, the LB of the MSE between $\boldsymbol{\alpha}_{l}$ and $\hat{\boldsymbol{\alpha}}_{\mathrm{des}_{l}}$ is given by:

$$
\mathrm{LB}_{l}=\mathrm{MSE}_{\mathrm{des}_{l}}+\mathcal{G} \times[\mathbf{S C R B}]_{l, l}
$$

In our case, the estimator of $\boldsymbol{a}$ is the LS-estimator (i.e., $\hat{\mathbf{A}}=$ $\mathbf{A}_{L S}$ ). Hence, (18) and (19) become:

$$
\begin{aligned}
& \mathrm{MSE}_{l}= \mathrm{MSE}_{\mathrm{des}_{l}}+\frac{1}{v N_{c}} \mathbf{g}_{l}^{H} \mathbf{R} \mathbf{g}_{l} \\
&-\frac{2}{v N_{c}} \operatorname{Re}\left(\mathrm{E}\left[\mathbf{e}_{\mathbf{d e s}_{l}}^{H} \mathbf{V} \boldsymbol{\Delta}_{\mathbf{p}}^{T}\right] \mathbf{g}_{l}\right) \\
& \text { with } \mathbf{R}= \mathrm{E}\left[\boldsymbol{\Delta}_{\mathbf{p}}^{*} \mathbf{V}^{H} \mathbf{V} \boldsymbol{\Delta}_{\mathbf{p}}^{T}\right]+\sigma^{2}\|\mathbf{V}\|^{2} \mathbf{I}_{N_{p}} \\
& \mathrm{MSE}_{l} \text { (without ICI) }=\mathrm{MSE}_{\operatorname{des}_{l}}+\frac{\|\mathbf{V}\|^{2}\left\|\mathbf{g}_{l}\right\|^{2}}{v N_{c} S N R}
\end{aligned}
$$

where $\boldsymbol{g}_{l}^{T}$ is the $l$ th row of the matrix $\boldsymbol{G}$. It is easy to show that:

$$
\left\{\begin{array}{l}
\mathrm{MSE}_{l}(\text { with ICI })>\mathrm{LB}_{l} \\
\mathrm{MSE}_{l} \text { (without ICI) }=\mathrm{LB}_{l}
\end{array}\right.
$$

So, by iteratively estimating and removing the ICI $\mathrm{MSE}_{l}$ will be closer to $\mathrm{LB}_{l}$.

\section{Simulation Results}

In this section, we verify the theory by simulation and we test the performance of the iterative algorithm. The normalized channel model is Rayleigh as recommended by GSM Recommendations 05.05 [12] [13], with parameters shown in Table I. A 4QAM-OFDM system with normalized symbols, $N=128$ subcarriers, $N_{g}=\frac{N}{8}$ subcarriers, $N_{p}=16$

TABLE I

PARAMETERS OF CHANNEL

\begin{tabular}{|c|c|c|}
\hline \multicolumn{3}{|c|}{ Rayleigh Channel } \\
\hline Path Number & Average Power $(d B)$ & Normalized Delay \\
\hline 1 & -7.219 & 0 \\
\hline 2 & -4.219 & 0.4 \\
\hline 3 & -6.219 & 1 \\
\hline 4 & -10.219 & 3.2 \\
\hline 5 & -12.219 & 4.6 \\
\hline 6 & -14.219 & 10 \\
\hline
\end{tabular}

TABLE II

THE GAIN $\mathcal{G}$ IN (21) WITH $N=128$ AND $N_{g}=16$

\begin{tabular}{|c|c|c|c|}
\hline$N_{c}$ & 2 & 3 & 4 \\
\hline $\mathcal{G}$ & 1.17 & 1.39 & 1.73 \\
\hline
\end{tabular}

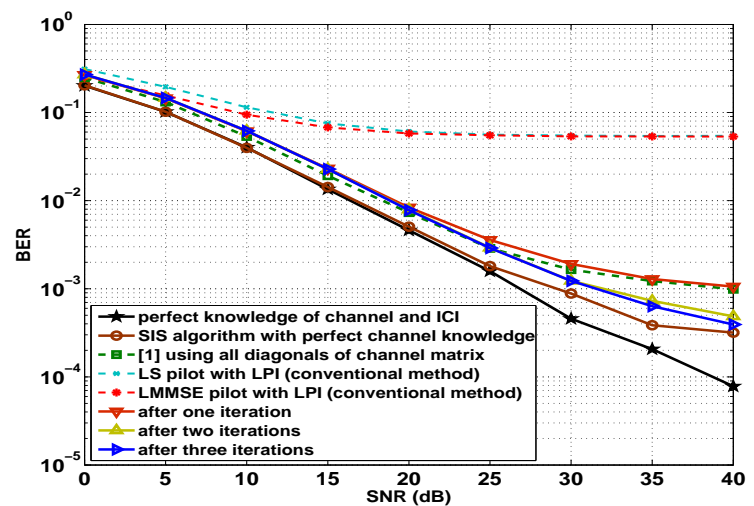

(a)

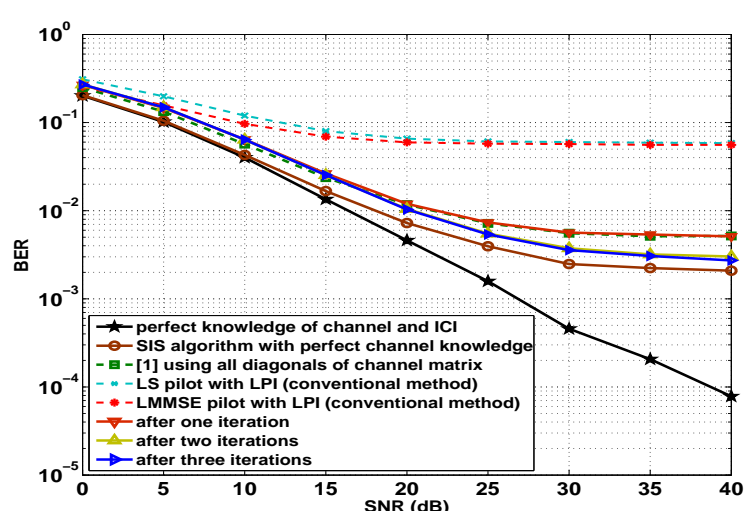

(b)

Fig. 5. BER for $N_{c}=2$ : (a) $f_{d} T=0.05$; (b) $f_{d} T=0.1$

pilots (i.e., $L_{f}=8$ ) and $\frac{1}{T_{s}}=2 M H z$ is used. (note that $\left.(S N R) d B=\left(\frac{E_{b}}{N_{0}}\right) d B+3 d B\right)$. These parameters are selected in order to be (with a scale factor) in concordance with the standard Wimax IEEE802.16e. The BER performance is evaluated under a relatively rapid time-varying channel such as $f_{d} T=0.05$ and $f_{d} T=0.1$ corresponding to a vehicle speed $V_{m}=140 \mathrm{~km} / \mathrm{h}$ and $V_{m}=280 \mathrm{~km} / \mathrm{h}$, respectively, for $f_{c}=5 G H z$.

Fig. 4 shows the evolution of MSE with the iterations in terms of SNR for $f_{d} T=0.1$. It is observed that, with all ICI, the MSE obtained by simulation agrees with the theoretical value of MSE. After one iteration, a great improvement is realized and MSE is very close to LB of our algorithm especially in low and moderate SNR regions. This is because at low SNR, the noise is dominant with respect to the ICI level and, at high SNR ICI is not completely removed due to the data symbols detection error. Fig. 4 also shows that, for $f_{d} T=0.1$ and $S N R \leq 30 \mathrm{~dB}$, the MSE of the polynomial approximation $\mathrm{MSE}_{\mathrm{des}}$ is negligible and the main contribution of the MSE is due to the LS-estimator. In this case, we indeed have from (21) that $\mathrm{LB}_{l} \approx \mathcal{G} \times[\mathbf{S C R B}]_{l, l}$ since $\mathrm{MSE}_{\mathrm{des}}$ is negligible when compared to SCRB. So, to find the smallest possible LB we have to choose $N_{c}=2$, since $\mathcal{G}$ increases in terms of $N_{c}$ as shown in Table II. However, at high SNR, LB is asymptotic to $\mathrm{MSE}_{\mathrm{des}}$ thus, the smallest possible LB will be with $N_{c}>2$ (see Fig. 3).

Fig. 5 gives the BER performance of our algorithm for 


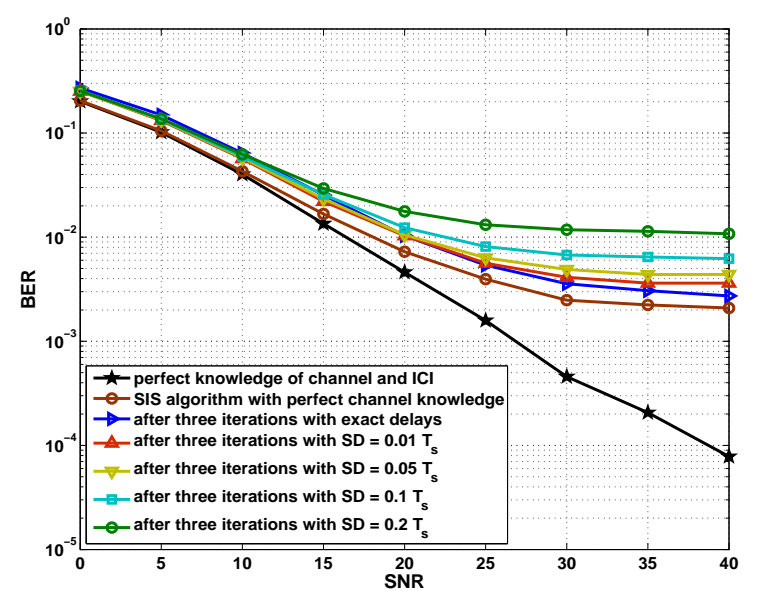

Fig. 6. Comparison of BER, for the case of imperfect knowledge of delays, for $N_{c}=2$ and $f_{d} T=0.1$

$N_{c}=2$, compared to conventional methods (LS and LMMSE criteria with low-pass interpolation (LPI) in frequency domain) [8] [9], our algorithm in [1], and SIS algorithm with perfect channel knowledge for $f_{d} T=0.05$ and $f_{d} T=0.1$. As reference, we also plotted the performance obtained with perfect knowledge of channel and ICI. This result shows that our algorithm performs better than the conventional methods and than our algorithm in [1]. Moreover, our iterative algorithm offers an improvement in BER after each iteration because the estimation of ICI is improved during each iteration. After two iterations, a significant improvement occurs; the performance of our algorithm and the SIS algorithm with perfect channel knowledge are very close. At a high SNR, it is normal to not reach the performance obtained with perfect knowledge of channel and ICI because we have an error floor due to the data symbol detection error. This error floor could be decreased by using a detection scheme better thant the SIS scheme.

Fig. 6 gives the BER performance after three iterations of our proposed iterative algorithm, for $N_{c}=2$ and $f_{d} T=0.1$, with imperfect delay knowledge. SD denotes the standard deviation of the time delay errors (modeled as zero mean Gaussian variables). It can be noticed that the algorithm is not very sensitive to a delay error of $\mathrm{SD}<0.1 T_{s}$. By using the ESPRIT method [10] to estimate the delays, we have a $\mathrm{SD}<0.05 T_{s}$, for all SNR as shown in Fig. 7. When combined with the ESPRIT method, our algorithm thus has negligible sensitivity to delay errors.

\section{CONCLUSION}

In this paper, we have presented a low-complexity iterative algorithm to estimate polynomial's coefficents of multipath complex gains and mitigate the inter-sub-carrier-interference (ICI) for OFDM system. The rapid time-variation complex gains are tracked by exploiting that the delays are assumed invariant (over several symbols) and perfectly estimated. Theoretical analysis and simultion results of our iterative algorithm show that by estimating and removing the ICI at each iteration, multipath complex gains estimation and coherent demodulation can have a great improvement especially after the first

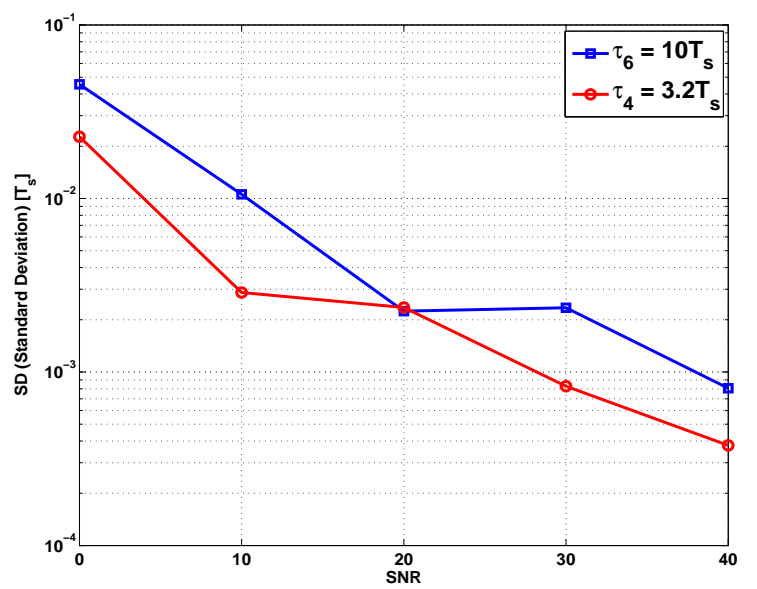

Fig. 7. Delay estimation errors for the fourth and sixth paths, using the ESPRIT method [10] (estimated correlation matrix, averaged over 1000 OFDM symbols, i.e $0.072 \mathrm{sec}$ ), for $f_{d} T=0.1$

iteration for high Doppler spread. Moreover, our algorithm performs better than the conventional methods and its BER performance is very close to the performance of SIS algorithm with perfect channel knowledge.

\section{REFERENCES}

[1] H. Hijazi, L. Ros and G. Jourdain, “ OFDM Channel Parameters Estimation used for ICI Reduction in time-varying Multipath channels" in EUROPEAN WIRELESS Conf., Paris, FRANCE, April 2007.

[2] E. Simon, L. Ros and K. Raoof," Synchronization over rapidly timevarying multipath channel for CDMA downlink RAKE receivers in TimeDivision mode",in IEEE Trans. Vehicular Techno., vol. 56. no. 4, Jul. 2007

[3] Z. Tang, R. C. Cannizzaro, G. Leus and P. Banelli, "Pilot-assisted timevarying channel estimation for OFDM systems" in IEEE Trans. Signal Process., vol. 55, pp. 2226-2238, May 2007.

[4] S. Tomasin, A. Gorokhov, H. Yang and J.-P. Linnartz, "Iterative interference cancellation and channel estimation for mobile OFDM" in IEEE Trans. Wireless Commun., vol. 4, no. 1, pp. 238-245, Jan. 2005.

[5] W. C. Jakes, Microwave Mobile Communications. Piscataway, NJ: IEEE Press, 1983

[6] Wikipedia contributors, "Linear regression", Wikipedia, The Free Encyclopedia.

[7] A. R. S. Bahai and B. R. Saltzberg, Multi-Carrier Dications: Theory and Applications of OFDM: Kluwer Academic/Plenum, 1999.

[8] M. Hsieh and C. Wei, "Channel estimation for OFDM systems based on comb-type pilot arrangement in frequency selective fading channels" in IEEE Trans. Consumer Electron., vol.44, no. 1, Feb. 1998.

[9] S. Coleri, M. Ergen, A. Puri and A. Bahai, "Channel estimation techniques based on pilot arrangement in OFDM systems" in IEEE Trans. Broad., vol. 48. no. 3, pp. 223-229 Sep. 2002.

[10] B. Yang, K. B. Letaief, R. S. Cheng and Z. Cao, "Channel estimation for OFDM transmisson in mutipath fading channels based on parametric channel modeling" in IEEE Trans. Commun., vol. 49, no. 3, pp. 467-479, March 2001.

[11] R. Roy and T. Kailath, "ESPRIT-Estimation of signal parameters via rotational invariance techniques" in IEEE Trans. Acoust., Speech, Signal Processing, vol. 37, pp. 984-995, July 1989.

[12] European Telecommunications Standards Institute, European Digital Cellular Telecommunication System (Phase 2); Radio Transmission and Reception, GSM 05.05, vers. 4.6.0, Sophia Antipolis, France, July 1993.

[13] Y. Zahao and A. Huang, " A novel channel estimation method for OFDM Mobile Communications Systems based on pilot signals and transform domain processing" in Proc. IEEE 47th Vehicular Techno. Conf., Phonix, USA, May 1997, pp. 2089-2093.

[14] H. L. Van Trees, Detection, estimation, and modulation theory: Part I, Wiley, New York, 1968. 